\begin{tabular}{|l|c|c|c|}
\hline $\begin{array}{l}\text { ACTA CLASSICA } \\
\text { UNIV. SCIENT. DEBRECEN. }\end{array}$ & LII. & 2016. & \\
\hline
\end{tabular}

\title{
NOTICES EPIGRAPHIQUES ET ONOMASTIQUES (DACIE ROMAINE) (I)
}

\author{
PAR DAN DANA
}

\begin{abstract}
This paper republishes 12 Greek and Latin inscriptions from Roman Dacia, in most cases with illustrations. Previous readings are improved and more ghost-names are removed. These inscribed monuments and objects (some of them, in the category of instrumentum inscriptum) are explained in their series or contexts, pertaining to the military milieu or the cosmopolitan side of the province.

Keywords: Greek and Latin epigraphy, instrumentum inscriptum, onomastics, Roman Dacia.
\end{abstract}

Je commence ici une série de notices épigraphiques et onomastiques concernant l'épigraphie latine et grecque de la province de Dacie, en améliorant la lecture et la compréhension de documents connus depuis longtemps ou récemment publiés ${ }^{1}$. La documentation photographique à laquelle j'ai eu accès permet de réviser la lecture de plusieurs monuments pour lesquels les lectures et les explications antérieures soulevaient des doutes; quelques inscriptions sont republiées ici pour la première fois avec une photographie $(\mathbf{3}, \mathbf{4}, \mathbf{1 2})$. Ces notices concernent des inscriptions grecques $(\mathbf{1}, \mathbf{7}, \mathbf{1 0})$, des inscriptions sur instrumentum $(\mathbf{3}, \mathbf{4}, \mathbf{6}, \mathbf{8}, \mathbf{9}, \mathbf{1 0})$, la correction de noms fantômes $(\mathbf{1}, \mathbf{2}, \mathbf{5}, \mathbf{9}, \mathbf{1 1})$ ou une meilleure compréhension d'inscriptions éditées $(1,3,4,6,7,8,9,10,12)$.

\footnotetext{
${ }^{1}$ Certaines de ces enquêtes sont en rapport avec mon ouvrage Onomasticon Thracicum (OnomThrac). Répertoire des noms indigènes de Thrace, Macédoine Orientale, Mésies, Dacie et Bithynie, Athènes, 2014 (MELETHMATA 70) ou avec le projet Epigraphica Romana du Centre ANHIMA (Paris). J'ai commencé ailleurs une série générale de «Notices onomastiques et épigraphiques » : Dana 2014a (= NEO I) ; Dana 2014b (= NEO II) ; Dana 2016 (= NEO III) ; une autre série concerne la Scythie Mineure (Dana 2014c). Pour des renseignements, conseils et permissions d'utiliser des images, j'exprime ici toute ma gratitude à Radu Ardevan (Cluj), Alexandru Avram (Le Mans), Gică Băeştean (Sarmizegetusa), Oana Bărbat (Deva), Cristina Bodo (Deva), Dorel Bondoc (Craiova), Dan Deac (Zalău), Răzvan Mateescu (Cluj), Ioan Piso (Cluj), Florian Matei-Popescu (Bucarest), Jean-Baptiste Yon (Lyon), Radu Zăgreanu (Bistrița), Karoline Zhuber-Okrog (Vienne).
}

doi $10.22315 / \mathrm{ACD} / 2016 / 8$ 


\section{Dédicace grecque de Sucidava $($ SEG XXIX $697=C I G D 121=I L D$ 112)}

Sur une plaque votive fragmentaire figurant le «Cavalier Thrace », découverte en remploi à Sucidava (Dacie Inférieure), fut gravée une dédicace grecque de deux lignes ${ }^{2}$. C. C. Petolescu préféra reprendre, en majuscules, la restitution douteuse du premier éditeur, Dumitru Tudor ${ }^{3}$, 'А то $\lambda(\lambda) \varepsilon v a ́$ pis $\Theta \varepsilon[\circ] l v o ́ k s$

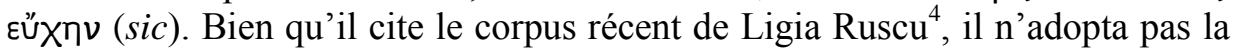
lecture correcte de cette dernière ${ }^{5}$, qui avait suivi la rectification de Georgi Mihailov $^{6}$ envoyée per litteras aux rédacteurs du SEG. Pour l'épigraphiste bulgare, le formulaire de la dédicace était banal, tel qu'on le rencontre à d'innombrables reprises en Thrace et en Mésie Inférieure. La lecture correcte est en effet celle retenue dans $S E G$ XXIX 697 :

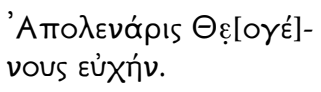

Dans la graphie du nom du dédicant, on remarque la simplification de la géminée (phénomène banal) et l'ouverture par dissimilation du -i- en -e- ${ }^{7}$. Pour ce dernier aspect, on peut renvoyer au nom du martyr Saint Apollinaire, écrit sanctus/beatus Apolenaris dans quelques inscriptions de Ravenne (CIL XI 293295), ou à la graphie du cognomen de Iulius Apollenaris à Ostie ${ }^{8}$. Sur le relief retrouvé à Sucidava, le choix de la langue grecque suggère qu'Apolenaris (= Apollinaris) fils de Théogénès était originaire du Sud du Danube.

\section{Le cognomen Gavianus (ILD 108)}

Sur un fragment de plaque votive de Sucidava (découvert toujours en remploi), figurant peut-être Némésis, le nom du dédicant a été lu Aur. Cavianus par le premier éditeur, Dumitru Tudor. Cette lecture, occasionnée d'ailleurs par une

\footnotetext{
${ }^{2}$ J'ai déjà suggéré cette correction dans le c.r. du répertoire $I L D$ (Dana 2010, 203).

3 Tudor 1979, 306-307, $\mathrm{n}^{\circ} 3$ (dessin p. 304, fig. 1.3 ; photo p. 306, fig. 3) :

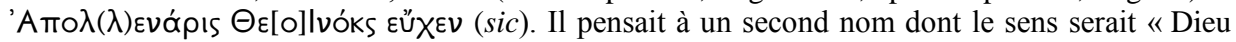
de la nuit », ce qui est pour le moins fantaisiste.

${ }^{4}$ CIGD 121 (photo Pl. XXIII), avec la transcription $\Theta \varepsilon[---]$ vous et la date correcte (II ${ }^{\mathrm{e}}$ s. ap. J.-C.).

${ }_{6}^{5}$ Petolescu ne faisait en réalité que reprendre sa notice CronEpigrRom I (1981) 34.

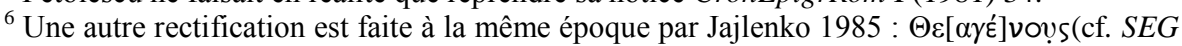
XXXV 856).

${ }^{7}$ Pour ces phénomènes dans l'espace hellénophone de Thrace et de Mésie Inférieure, voir Slavova 2004, 113 (simplification de géminées liquides) et 22 (ouverture par dissimilation).

${ }^{8}$ Thylander 1952, n $^{\circ}$ A 137.
} 
explication douteuse ${ }^{9}$, a été adoptée par C. C. Petolescu (ILD 108) : Aur(elius) Cavianus ex $\mid$ votu posuit. Certaines lettres de l'inscription sont cursives (ainsi, le S). Un dérivé Cavianus, tiré d'un gentilice très rare Cavius, est pourtant très rare $^{10}$, et peu probable en association avec le gentilice impérial qui nous situe après 212. Dans la dédicace de la ville dace, il convient plutôt de lire Aur. Gavianus, cognomen dérivé du gentilice fréquent Gavius - utilisé auparavant comme idionyme ${ }^{11}$. Depuis peu, on connaît un toponyme hybride latino-gète dans le voisinage de Durostorum, en Mésie Inférieure : vicus Gavidina, «village de Gavius » ${ }^{12}$.

\section{Une marque de propriété militaire sur une pièce de bronze d'Vlpia Sarmizegetusa (ILD 291)}

Il y a plus de deux décennies, Adela Paki publiait une marque de propriété sur instrumentum provenant d'Vlpia Sarmizegetusa, avec un dessin mais sans aucune photo. Ce qui était décrit comme un «bouton de bronze » est en réalité un disque circulaire de bronze (diam. 3,4 cm ; ép. 0,1-0,5 cm), découvert en 1979 lors des fouilles dans le forum, dans la partie sud de la cour, probablement dans la couche 9. Il présente une inscription circulaire en pointillé (ht. des lettres $0,7-$ $0,8 \mathrm{~cm}$; interponction), autour du clou détachable de bronze qui permettait sa fixation (sur un support en cuir ?) sur une arme. La lecture de Paki était ${ }^{13}$ : [T(urma)] Veri. M(arcus) Carte[ius] ou M(arci) Carte[i Se]veri ; elle privilégiait la dernière variante, étant suivie par C. C. Petolescu (ILD 291). Une meilleure lecture et compréhension furent données par Ioan Piso en 2006, qui précise pourtant que la pièce est « introuvable» :M(arci) Cartei ?t(urma) Severi ${ }^{14}$.

\footnotetext{
${ }^{9}$ Tudor 1981, 434-436, $\mathrm{n}^{\circ} 34$ (avec photo peu lisible p. 432, fig. 7.2 ; et dessin p. 435, fig. 11.1) (= $A E ́$ 1981, 720) ; toujours à la recherche d'étymologies, il voyait en Cavianus le «Gardien », ce qui est impossible ; C. C. Petolescu, CronEpigrRom II (1981-1982), 107 ; HD 005796. Malgré mes efforts, je n'ai obtenu aucune réponse de la part du Musée de Corabia, où l'objet est conservé.

${ }^{10}$ Pinarius Cavianus à Segusio, dans les Alpes Cottiennes (AÉ 2007, 893) ; Geminia Caviana à Luna, en Étrurie $(A E ́$ 1998, 435).

${ }^{11}$ Solin 2010, 235, évoque la possibilité de corriger en Gavianus, tout en mentionnant un cognomen Cavianus. J'avais déjà suggéré cette correction dans le c.r. au répertoire ILD (Dana 2010, 203).

$12 A E ́$ 2011, 1137 = ISM IV 93 ; voir Boyanov 2010. Cf., dans la même province, le toponyme Longinopara, « village de Longinus » (ILBulg 209).

${ }^{13}$ Paki 1989-1993, 297, nº 6 (dessin p. 296, Pl. III.3) ; $A \dot{E}$ 1993, 1348, avec la lecture [T(urma)] Veri M. Carte[ius] ; ILD 291 ; HD 043768.

${ }^{14}$ Piso 2006, 313-314, n ${ }^{\circ} 106$ (et dessin, fig. III.103 : tessera en bronze, avec un umbo) $(=A E ́$ 2006, 1172 : M. Cartei ț(urma?) Șeveri). Pour ce type de marques de propriété, voir la bibliographie donnée dans la notice $n^{\circ} 4$.
} 
Piso identifie ainsi le nom de l'officier, Severus (à la place de Verus), tout en précisant qu'il n'y a aucune « certitude pour t(urma); l'unité peut être out aussi bien une (centuria) ».

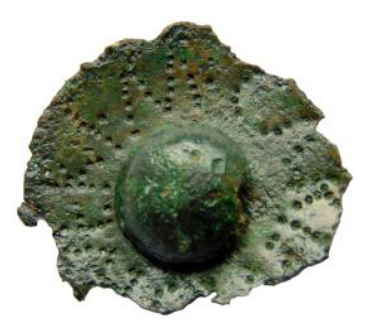

Fig. 1. Photo de la plaquette de bronze (C) Mus. Sarmiz. inv. 24458, cliché G. Băeștean)

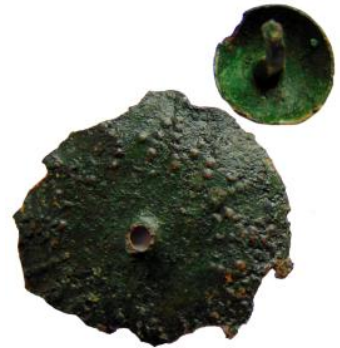

Fig. 2. Photo de la plaquette de bronze (verso), avec son clou détachable (C) Mus. Sarmiz. inv. 24458, cliché G. Băeştean)

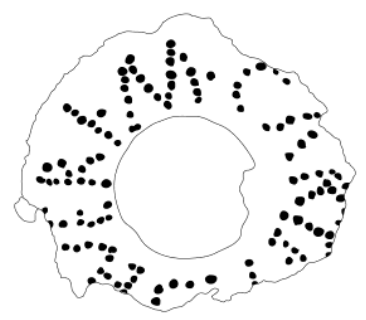

Fig. 3. Fac-similé de la plaquette de bronze (D. Dana)

L'objet, conservé dans le musée local (Mus. Sarmiz. inv. 24458), n'est pas perdu, et n'est certainement pas un élément d'un bouclier. On peut donner pour la première fois des photos des deux faces de la plaquette de bronze (avec et sans le clou détachable), puisque sur le verso on aperçoit, en négatif, les traces des lettres en pointillé, qui aident à confirmer la lecture du recto. On lira $\mathrm{M}$ • CARȚEI $[\bullet]+$ ṢEVERI, ce qui donne :

\section{M(arci) Carței, ț(urma)/(centuria) Șeveri.}

L'objet fut perdu vers la fin des guerres daciques, au moment où le site était occupé par des détachements de la legio IV Flavia et sans doute d'unités auxiliaires. Le soldat est un citoyen romain, identifié par ses duo nomina, M. Carteius. Il est porteur d'un gentilice très rare ${ }^{15}$, attesté par hasard vers l'époque de Commode dans la même province : Carteia Maxima, femme de C. Iul. Frontonianus, vet(eranus) ex b(ene)f(iciario) co(n)s(ularis) leg(ionis) $V$, qui dédie à Apulum un autel à Esculape et Hygie (CIL III $987=I L S 3847=I D R$ III.5 21).

Avant le nom de l'officier, par ailleurs très banal (Severus), la pièce est abîmée et ne permet pas de trancher entre les seules possibilités, à savoir la lettre $\mathrm{T}$ (mention de l'escadron) ou le signe 7 pour centuria. Comme sur la plupart des autres marques de propriété des militaires (voir infra, 4), le nom du soldat est au génitif.

\footnotetext{
${ }^{15}$ Solin - Salomies 1994, 48.
} 


\section{Relecture d'une tessera militaris de Hoghiz (IDR III.4 244)}

En 1949, lors des fouilles dans le camp auxiliaire de Hoghiz (dép. de Braşov), plus précisément en son milieu, fut découvert un disque de bronze. Dimensions : $3,41 \times 3,27 \times 0,6 / 0,7 \mathrm{~cm}$; poids : $2,32 \mathrm{gr}$. Le disque est perforé au milieu et son orifice carré mesure 2,7 x 2,7 mm. Cluj, MNIT (inv. IN 3317). Après une publication préliminaire par K. Horedt avec un dessin, une transcription en majuscules et une proposition d'interprétation de I. I. Russu, l'inscription en pointillé fut éditée avec le même dessin dans l'un des fascicules du corpus de la province, par l'érudit I. I. Russu, avec la lecture suivante ${ }^{16}: T(i t i)$ Aviti | [Ge]rmani. Récemment, à l'occasion de la publication d'une tessera militaris d'Arcobara/Ilișua et d'une étude sur la présence militaire sur le limes oriental de la Dacie (en Transylvanie), la lecture fut émendée ${ }^{17}$, mais sa republication s'impose.

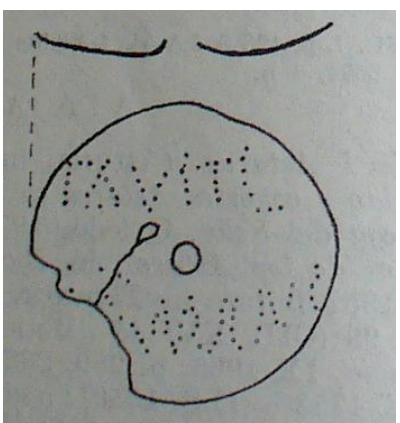

Fig. 4. Dessin de la tessera militaris (MCA 1, 1953, 792, fig. 12.4 ; IDR III.4, p. 192 , fig. 148)

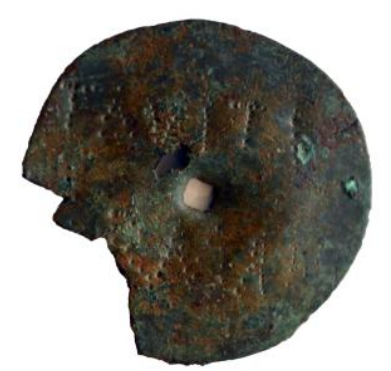

Fig. 5. Photo de la tessera militaris (C) MNIT inv. IN 3317 , cliché R. Mateescu)

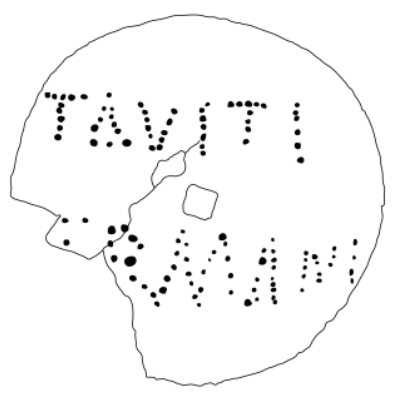

Fig. 6. Fac-similé de la tessera militaris (D. Dana)

Entre les deux lignes de texte, avec des lettres en pointillé (ht. 0,5-0,6 cm), se trouve l'orifice central rectangulaire, grâce auquel le disque était attaché (à une arme ?) ; le tout début de la deuxième ligne est perdu et endommagé. I. I. Russu donna dans IDR la lecture T(iti) Aviti | [Ge]rmani, comme s'il s'agissait d'un citoyen romain «T. Avitius Germanus »; à la place du praenomen, F. Matei-Popescu choisit de lire (centuria) Aviti, en mettant en rapport cette mention avec la coh. III Gallorum, attestée à Hoghiz ${ }^{18}$. Pourtant, sur le dessin (IDR

\footnotetext{
${ }^{16}$ Horedt 1953, 792 et n. 12 (et fig. 12.4) ; IDR III.4 244 ; HD 045430.

${ }^{17}$ Dana-Gaiu 2014, 156 et n. 22 ; Matei-Popescu 2014, n. 70 à la p. 214.

${ }^{18}$ Petolescu 2002, 106-107.
} 
III.4, p. 192) il était possible d'apercevoir la lettre T, abréviation de t(urma). Autant dire que la vérification sur l'original s'imposait.

On peut donner pour la première fois une photo de l'objet, avec un facsimilé plus fidèle, et une lecture assurée :

T(urma) Aviti,

[G]ermani.

Datant vraisemblablement du $\mathrm{II}^{\mathrm{e}}$ s., cette tessera militaris décline le nom et l'escadron (identifié d'après le décurion Avitus) d'un eques de l'ala I Asturum ou de la vexillatio equitum Illyricorum, unités présentes dans le camp auxiliaire de Hoghiz ${ }^{19}$, en Dacie Inférieure ${ }^{20}$.

Ce type de tessera militaris s'inscrit dans la série des procédures d'identification ${ }^{21}$ ou des marques de propriété des armes ${ }^{22}$. Rectangulaires ou circulaires, avec des orifices qui permettaient de les attacher, ces plaquettes indiquaient la sous-unité du soldat : soit la centurie (dans les cohortes auxiliaires et les légions), soit la turme (dans les ailes). On connaît un dossier assez consistant de tesserae militares en Dacie romaine, la dernière en date provenant du camp de l'ala I Tungrorum Frontoniana (à Arcobara/Ilişua) : une applique de bronze en forme de tabula ansata, avec une inscription en pointillé, appartenant à l'eques Marcellus, de la turme de Ianuarius ${ }^{23}$.

\footnotetext{
${ }^{19}$ Petolescu 2002, 62-63 et 131-132.

${ }^{20}$ Disparait ainsi la seule occurrence en Dacie du rare gentilice Avitius (OPEL I ${ }^{2}$ 97), au profit du fréquent idionyme/cognomen Avitus (OPEL $\mathrm{I}^{2}$ 97-98). À son tour, Germanus est banal (OPEL II 166). La seule autre possibilité de lecture de la 1. 2 est moins plausible : Fịrmani.

${ }^{21}$ Cosme 2007.

${ }^{22}$ Voir, entre autres, MacMullen 1960 ; Nuber 1972 (et catalogue de 65 marques de propriété, p. 504-507) ; Oldenstein 1976, 190-193 (Pl. 60-61) ; Schmidt Heidenreich 2012, 396. Pour ce type d'inscriptions, voir RIB II.1 2410.1-8 et 1223 (Bretagne) ; de très nombreux exemples chez Pfahl 2012 (= ILGIL), 170-175, $\mathrm{n}^{\text {os }}$ 241-319 (Pl. 36-39) (sur le limes du territoire de l'actuelle Allemagne). Un lot de pièces présentes dans le commerce d'antiquités vient d'être publié par Eck - Pangerl 2015.

${ }^{23}$ T(urma) Ian(u)ari,| Marcel|ḷ ++. Voir Dana - Gaiu 2014, 155-157, n 1 (= Dana - Gaiu 2016, 266-267), avec d'autres exemples connus en Dacie. La plupart des tesserae militares de Buciumi viennent d'être republiées, avec un meilleur déchiffrement, par Piso 2015, 209-212, $\mathrm{n}^{\text {os }}$ 29-31 (coh. I Brittonum milliaria et coh. I Flavia Vlpia Hispanorum milliaria civium Romanorum equitata).
} 


\section{Sur un nom iranien à Apulum (IDR III.5 707)}

Un autel votif découvert en 1986 dans le lit de la rivière Mureş fut édité de la manière suivante ${ }^{24}$ et repris tel quel dans le corpus de la province (IDR III.5 707) - Farna|c?an(e)s Ga[i] | Mart[i] $\mid$ d(ono) d(edit) [d(edicavitque)] - ainsi que dans toutes les autres références, à l'exception de S. Sanie, qui proposa une lecture très fantaisiste ${ }^{25}$. Il serait toutefois étonnant que le nom même du dédicant soit écrit de cette manière, en supposant une ligature AN et une lettre oubliée ou à peine esquissée $(\mathrm{E})^{26}$ pour un nom qui, en outre, est hapax (un nom dérivé ou composé du nom iranien Фapvóknऽ). La solution la plus facile est d'interpréter les deux hastes verticales du début de la 1.2 , après le $C$, comme le signe bien connu pour la lettre $\mathrm{E}^{27}$. Remarquons que cette face de l'autel a été endommagée, sans doute au moment de la récupération du monument du lit du Mureş. Cette trace, qui affecte également les lignes suivantes, ne peut pas cacher la haste oblique du $\mathrm{N}$ supposé par les éditeurs. On lira donc :
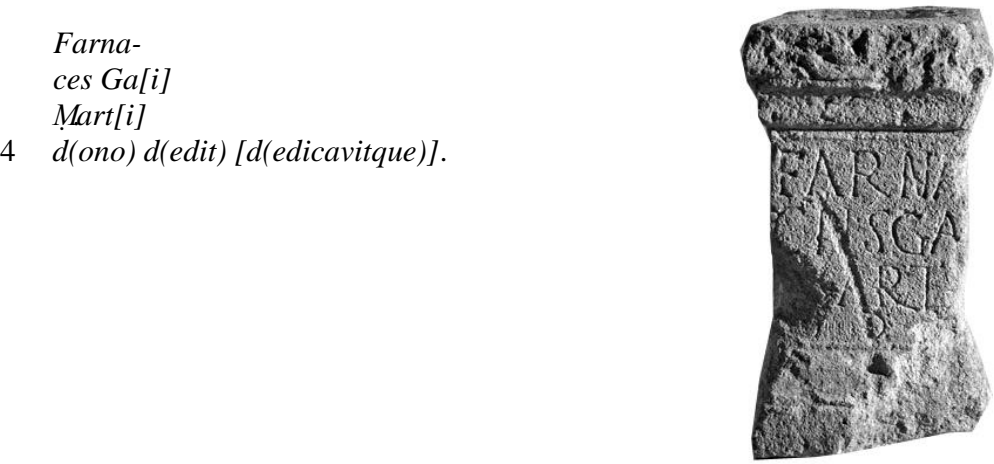

Fig. 7. Photo de l'autel d'Apulum

(C) MUAI inv. 9566, cliché R. Zăgreanu)

\footnotetext{
${ }^{24}$ Moga - Piso - Drîmbărean 1998, 111-113, nº 2 (photo p. 112, fig. 2 a ; dessin p. 113, fig. 3 A) (= AÉ 1998, 1078); C. C. Petolescu, CronEpigrRom XVIII (1998) 798 (même lecture) (=ILD 459 : « la lecture soulève certains points d'interrogation ») ; MNUAI, inv. 9566.

${ }^{25}$ Sanie 1999, 175-177, $\mathrm{n}^{\circ} 3$ (photo p. 172, fig. 1.4) : Farna[x] | cane(phoro)s gal|[lusque] Att [i] |d(ono)d(edit) [d(edicavitque)] (cf. AÉ 1999, 1294 : « lecture impossible : Mart [i] à la 1.3 est sûr »); C. C. Petolescu écrit avec raison [CronEpigrRom XIX-XX (1999-2000), 826 c] : «rectification fantaisiste, qui ne mérite même pas d'être rapportée ».

${ }^{26}$ De l'avis même de I. Piso, « une ligature ANI ou ANE est très peu sûre » (IDR III.5, p. 513) ; le dessin donné dans le corpus est légèrement différent de celui de la revue $A M N$. Une autre photo de l'autel : Lupa 12242.

${ }^{27}$ Pour un exemple récent, voir, à Alburnus Maior, le nom d'un Helius, transcrit HIILIVS

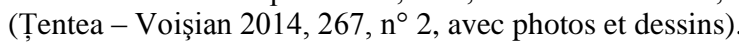


Farnaces est alors le nom iranien bien connu (grec Фapvókns) ${ }^{28}$, déjà attesté par deux fois en Dacie romaine : en 180, C. Iulius Farnax, Augustalis du municipium Aurelium Apulense (CIL III $986=$ IDR III.5 20) ; au III ${ }^{\mathrm{e}}$ s., le légionnaire Aur. Farnax à Potaissa (CIL III 7688). Notre Farnaces fils de Gaius, sans doute un ressortissant d'Asie Mineure (voir $\mathrm{n}^{\circ} 7$ ), s'était installé dans le centre cosmopolite d'Apulum au $\mathrm{II}^{\mathrm{e}} \mathrm{s}$.

\section{Un nom thrace dans un graffite d'Apulum (CIL III 1635,5 = IDR III.6 354)}

Un graffite réalisé ante cocturam dans la partie inférieure d'une céramique sigillée de type indéterminé (dimensions : 8,2 x 8,1 cm), découverte au XIX ${ }^{\mathrm{e}} \mathrm{s}$. à Apulum et entrée de la collection de Johann Michael Ackner au Kunsthistorisches Museum, Antikenkabinet de Vienne (inv. $n^{\circ}$ IV 1437 d), a été publié à plusieurs reprises, avec des dessins et des lectures divergentes ${ }^{29}$. Cloşca Băluță, qui donne un dessin similiare à celui du CIL (IDR III.6, p. 179, fig. 351), lit, d'après l'édition Ackner - Müller (1865), Drus[s]us f(ecit) ${ }^{30}$. Or, cette lecture présuppose une signature sinistroverse, qui est peu probable.

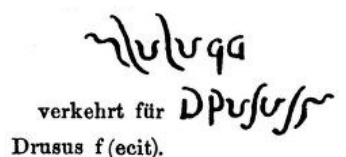

Fig. 8. Dessin M. J. Ackner (1865) (copie et interprétation)

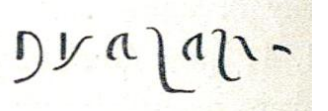

Fig. 9. Dessin CIL III 1635,5 (réproduit par C. Băluță, IDR III.6, p. 179, fig. 351)

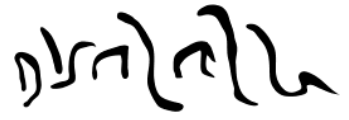

Fig. 10. Fac-similé (D. Dana)

L'accès à une documentation photographique du tesson m'a permis d'en faire un fac-similé. L'écriture est cursive ${ }^{31}$, notamment pour les lettres A, L, S (ht. max. des lettres : 1,2 cm) (fig. 10). Ma propre lecture est :

Disala $L V(?)$.

\footnotetext{
${ }^{28}$ Pour ses transcriptions en latin et ses occurrences à Rome, voir Solin 2003 I, 243-244.

${ }^{29}$ Ackner - Müller 1865, 112, n 539 (et dessin) ; CIL III 1635,5 (et dessin), sans transcription ; Cserni 1901, 536, $\mathrm{n}^{\circ} 17$.

${ }^{30}$ Falileyev 2007,85 , est enclin à prendre ce nom pour celtique (mais il note que « the restoration of the second $s$ is unnecessary ») et va plus loin : «note, however, the strong associations of terra sigillata found in Dacia with the production centre in Lezoux, Gaul », oubliant que dans ce cas précis il ne s'agit pas d'une marque de potier, mais d'un graffite.

${ }^{31}$ Pour la province qui nous intéresse, voir Voloşciuc 2007 (qui ne semble pas avoir pris en compte ni cet exemple, ni la brique de Gherla, cf. $^{\circ}{ }^{\circ} \mathbf{9}$ ).
} 
Il s'agit donc d'un nom thrace, variante connue du nom fréquent Dizala/ $\Delta \mathrm{L} \zeta \propto \lambda_{\alpha} \alpha_{S}$ (OnomThrac 151-152) ${ }^{32}$. Sans doute un militaire, notre Disala avait inscrit après son nom un chiffre.

\section{Un hellénophone de Galatie porteur d'un nom iranien à Alburnus Maior $($ IDR III.3 399 = CIGD 4)}

Un autel découvert en 1936 sur la colline Carpeni d'Alburnus Maior porte une inscription grecque ${ }^{33}$ :

$$
\begin{aligned}
& \triangle \eta \mu n \dot{T}- \\
& \text { plos ApS- } \\
& \text { वKध। } \triangle \varepsilon- \\
& 4 \text { i Napqu- } \\
& \text { ต̃ घưXń- } \\
& v \text {. }
\end{aligned}
$$

Il s'agit donc d'une dédicace à Zeus Narènos de la part de Dèmètrios, fils d'Arzakis. La patronyme du dédicant fut différemment lu et surtout différemment reconstitué au nominatif, avec un léger avantage pour $A \rho \zeta \alpha$ kns. Pourtant, vu la forme du génitif ( $A \rho \zeta \alpha \kappa \varepsilon l)$, un seul nominatif est possible: $A \rho \zeta \alpha \kappa(\varepsilon) ı$; on a donc affaire à un génitif asigmatique. $A \rho \zeta \alpha \mathrm{K}(\varepsilon)$ เs est évidemment une variante de Apođkns, nom iranien fréquent (qui donna le nom de la dynastie des Arsacides), mais qui présente ici une forme syncopée de la terminaison latine (-ius,-105) et, trait remarquable, la sonorisation de la sifflante (-s- > -z- $)^{34}$.

Non seulement cette dédicace entre dans le petit mais d'autant plus remarquable dossier épigraphique grec du site minier $(C I G D 3,5,7,8)$, mais le Zeus Narènos honoré ici comme dans une autre dédicaces d'Alburnus Maior (CIGD

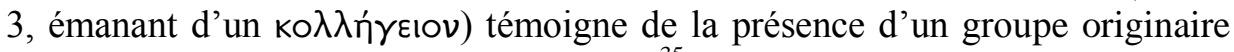
de Nara, toponyme non-localisé de Galatie ${ }^{35}$.

\footnotetext{
${ }^{32}$ Cette relecture est signalée dans OnomThracSuppl [151-152].

${ }^{33}$ IDR III.3 399 = CIGD 4. Dernière édition, avec l'ensemble de la bibliographie : Ciongradi 2009, 54-55, n 32 (photo Pl. 19).

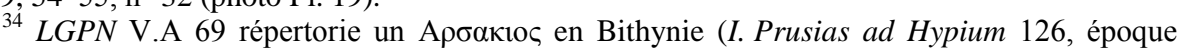
tardive) ainsi qu'un martyr, Arsakis de Nicomédie (Sozomène, $H E$ 4.16). Sous la forme syncopée Apoakıs, on rencontre ce nom dans une épitaphe chrétienne de Panticapée, au Nord de la mer Noire (IOSPE V 270); et, en Asie Mineure, à Kozanlı (voir dans le texte).

35 Pour cette divinité, voir Nemeti 2005, 249-252 ; cf. brièvement Schäfer 2009. Pour la localité galate, voir la notice désormais dépassée de Ruge 1935 (entre Tembrogios et le moyen

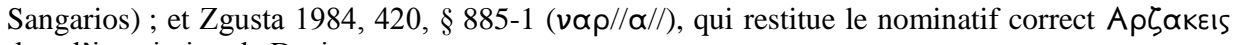
dans l'inscription de Dacie.
} 


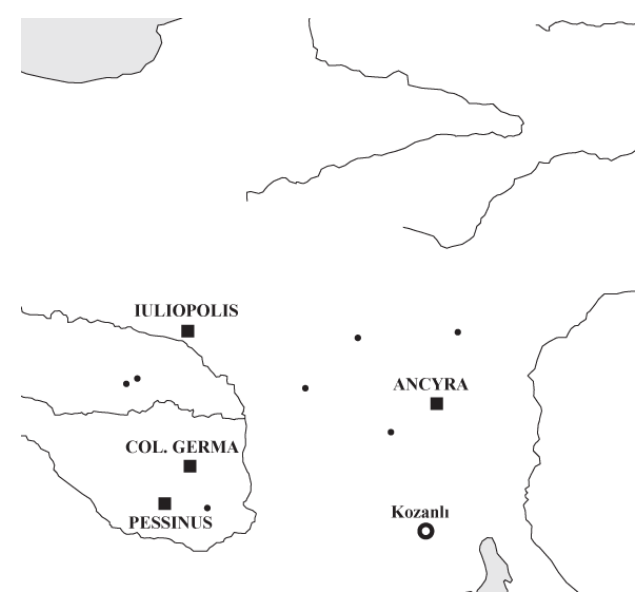

Fig. 11. Dédicaces à Zeus Narènos (Galatie).

Il convient d'insister sur le fait que plusieurs dédicaces à Zeus Narènos furent trouvées en Galatie même. Le corpus régional RECAM II comporte ainsi 7 dédicaces à cette divinité topique, dans une région assez vaste entre Iuliopolis, colonia Germa et Ancyra : à Beylikahir (11, 12), Yukari Dudaş (42), Güce (53), Mihalıççık (67), Yarıkcı (70) et Güreş (86). Sur le plan onomastique, la présence sporadique de noms iraniens en Galatie ou plus généralement en Asie Mineure est bien attestée ${ }^{36}$. Il est remarquable de trouver le même nom, Apođkıs, sous une forme syncopée, dans une épitaphe de Kozanlı (MAMA VII 442), aux confins de la Phrygie et de la Galatie, précisément au Sud de la région où l'on répertoire les dédicaces pour Zeus Narènos (voir la carte, fig. 11).

À côté des Bithyniens, particulièrement nombreux en Thrace, Mésies et Dacie, en raison notamment de leurs compétences techniques ${ }^{37}$, les ressortissants de la province de Galatie sont en outre attestés en Dacie romaine par des associations à caractère cultuel, autour de leurs divinités : un col(legium) Galatarum à Germisara (CIL III $1394=$ IDR III.3 234), ou encore des Gal[at]ae consistentes à Napoca, honorant IOM Tavianus (CIL III 860) ${ }^{38}$.

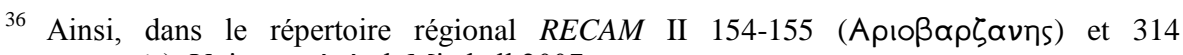
(Парvakns, sic). Voir, en général, Mitchell 2007.

${ }^{37}$ Voir Avram 2013.

${ }^{38}$ Pour un autre exemple de ces formes de solidarité religieuse, ethnique ou provinciale, cf. un collegium Ponto-Bithynorum à Apulum (IDR III.5 153), regroupant les personnes originaires de la province Pontus et Bithynia. Voir, en général, la synthèse de Mihăilescu-Bîrliba 2011 (sur les Galates, 14-15). Selon la formule judicieuse de Popescu 2004, 158, les communautés micrasiatiques en Dacie sont «largement minoritaires en terme démographique, mais actives sur le plan épigraphique ».
} 


\section{Lamelle d'or de Germisara avec un nom dace (ILD 325)}

Il y a deux décennies, la publication d'un lot de 7 lamelles d'or ${ }^{39}$, dont 5 inscrites, découvertes en 1987 lors des fouilles de sauvetage dans les bains antiques de Germisara (Geoagiu-Băi, dép. de Hunedoara) ${ }^{40}$, a occasionné la surprise de trouver parmi les dédicants un nom indubitablement dace ; cette découverte constituait à l'époque la première attestation certaine d'un nom indigène dans la province nord-danubienne. Qui plus est, il s'agit du même nom que celui porté par le dernier roi du royaume anéanti par la volonté de Trajan. Le texte donné par les éditeurs de cette plaquette $(16,2$ x 6,9 cm ; 13,53 gr.; ht. des lettres : 0,7 cm ; MCDR, inv. 34536), avec le champ épigraphique en forme de tabula ansata, était le suivant : Nymf|is Dece|balus Lu|ci posuit. En réalité, la vérification sur des photos de bonne qualité permet de corriger légèrement la lecture du nom du dédicant :

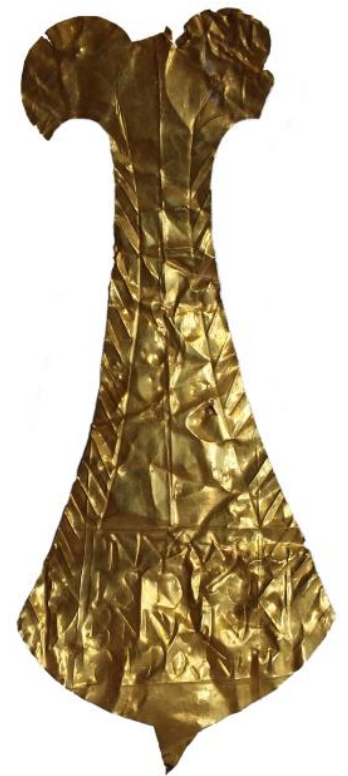

Fig. 12. Photo de la lamelle d'or (C) MCDR inv. 34536, cliché O. Bărbat)

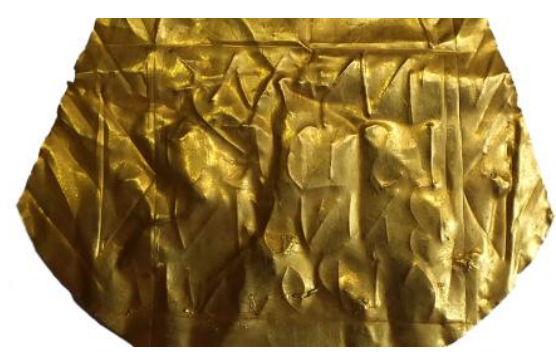

Fig. 13. Photo de la lamelle d'or (verso) (C MCDR inv. 34536, cliché O. Bărbat)

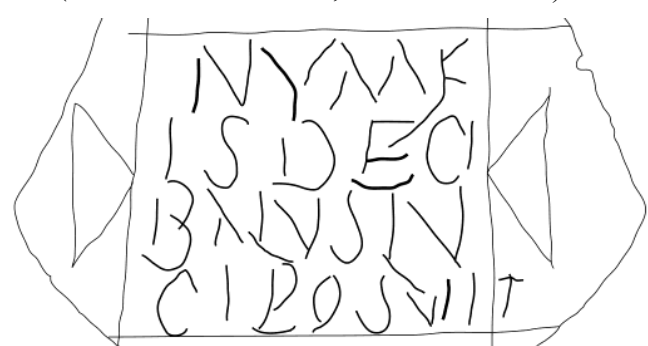

Fig. 14. Fac-similé de la lamelle d'or (D. Dana)

\footnotetext{
${ }^{39}$ Pour ce type de lamelles, voir en dernier lieu Weber 2012.

${ }^{40}$ Piso - Russu 1990, 12, n 5, fig. 10 (photo) et 11 (dessin) (= $A E$ 1992, 1483) ; C. C. Petolescu, CronEpigrRom XII (1992) 586 (= ILD 325); voir aussi Dana 2004, 438. Pour d'autres lamelles, découvertes vraisemblablement à la même époque mais entrées dans des collections privées, voir Ardevan - Cociş 2014.
} 


$$
\begin{array}{ll} 
& \text { Nymf- } \\
& \text { is Deci- } \\
\text { balus Lu- } & \text { ciposuit. }
\end{array}
$$

On lit en vérité Decibalus, ce qui est confirmé par la photo de l'autre face de la lamelle, où l'on observe nettement un I, à la place du E supposé par les premiers éditeurs. C'est d'ailleurs la forme présente dans les sources latines pour le nom du dernier roi dace ainsi que dans la plupart des mentions épigraphiques grecques et latines ${ }^{41}$ - en contraste avec la forme consacrée dans l'historiographie moderne, Decebalus/Décébale, sur la foi du texte de Dion Cassius. Malgré le débat sur le statut exact du porteur de ce nom à Germisara, dans la seconde moitié du $\mathrm{II}^{\mathrm{e}}$ s., en raison de sa formule onomastique - esclave, affranchi, pérégrin $?^{42}$, voire citoyen romain selon C. C. Petolescu ${ }^{43}$-, il s'agit le plus vraisemblablement d'un pérégrin, Decibalus fils de Lucius. On trouve par ailleurs une onomastique mélangeant noms daces et latins sur deux diplômes militaires accordés à des militaires daces en service en Dacie Porolissensis ${ }^{44}$ et en Dacie Inférieure ${ }^{45}$. Ce $«$ nom historique ${ }^{46}$ par excellence des Daces est à présent connu par environ 25 occurrences (OnomThrac 115-117). Pour un autre nom dace dans la province, voir la notice suivante.

\section{Une brique avec un exercice d'écriture à Gherla (CIL III 294*)}

Une brique découverte vraisemblablement en 1890, lors des fouilles, dans les substructions des thermes du camp auxiliaire de Gherla, porte une inscription fausse d'après Alfred von Domaszewski, qui avait reçu une photo de József Ornstein : « in tegula antiqua litterae noviciae exaratae sunt » (CIL III, p. 35*, 294*). Son caractère authentique a été pourtant démontré par les antiquisants hongrois et, à partir de I. I. Russu, par les éditeurs roumains. Il s'agit manifes-

${ }^{41}$ Si l'on laisse de côté le nom du roi, la documentation disponible offre les graphies suivantes : 15 fois Decibalus $/ \triangle \varepsilon \kappa / \beta \alpha \lambda$ os ; 7 fois Decebalus/ $\triangle \varepsilon \kappa \varepsilon \beta \alpha \lambda$ os ; 5 fois Dicebalus/ $\triangle \mathrm{ik \varepsilon} \beta \alpha \lambda$ os (voir OnomThrac 115-117).

${ }^{42}$ Protase 1995, 157-158 (dessin p. 163, fig. 1).

${ }^{43}$ Ce qui est très improbable, voir Dana 2010, 204.

${ }^{4} A E ́$ 2003, 2046 (ca. 133/140) : coh(ortis) II Aug(ustae) Nerv[ianae (milliariae) Pacensis (...) ex pe[dite] Didaecutti L[--- f(ilio), Daco?], et Diurpae Dotu[si? fil(iae) uxori eius, Dacae?], et Iulio f(ilio) [eius, et --- $f($ ilio)/fil(iae) eius], et Dimidusi fil(iae) [eius, ?et --- fil(iae) eius].

${ }^{45}$ RMD V 389 (ca. 120/140) : [coh(ortis) --- (...)], ex pedi[te ---] Lucii f(ilio), [---, et --f(ilio) eius, e]t Tarae $f($ ilio) eiu[s, et --- $f($ ilio)/fil(iae) eius, et ---a]e fil(iae) eius, e[t --- fil(iae) eius].

${ }^{46}$ Pour Decibalus comme « nom historique » des Daces, voir Dana 2006 (en partic. 114-116 et 121-124), 2007 (en partic. 43-45) et 2011, 79-82. 
tement d'un exercice d'écriture, incisé avant cuisson, et comportant deux séquences : a) liste de noms ; b) alphabet latin. Tuile carrée $(27,2 \times 27,2 \times 4,5 \mathrm{~cm})$ avec inscription cursive; ht. des lettres : 1,8-2 cm. Au Musée de Gherla (inv. 656). Lettres remarquables: A (sans haste médiane), G (avec un trait oblique vers la gauche), R (de forme ouverte), Z (qui a la forme d'un sigma à trois branches, après un gribouillage). Une lettre ( $\mathrm{T}$ ?) est décalée à gauche par rapport aux 1l. 3-4; $\mathrm{N}$ renversé au lieu de $\mathrm{A}^{47}$ et $\mathrm{A}$ sans haste médiane au lieu de $\mathrm{N}$ (1. 4). Quelques points de séparation (11. 1-3; dont deux points à la 1. 3).

CIL III *294 ; Fröhlich 1892 ; Ornstein 1904 (et photo) ; Finály 1906, 38-39, n V ; Russu 1964, 480-481, n 10 (dessin p. 480, fig. 1) ; Tudor 1965, 110 (photo, et mention p. 112) ; I. I. Russu, IDR III.2, 1980, p. 420 ; Gudea 1987, 101, n 33 (dessin p. 136) ; Protase - Gudea - Ardevan 2008, 51/215, no 49 (dessin Pl. VII) ; Falileyev 2009 (cf. $A E ́$ 2010, 1365 ; ILD 1007); Lupa 11393 (R. Ardevan) ; HD 072086.

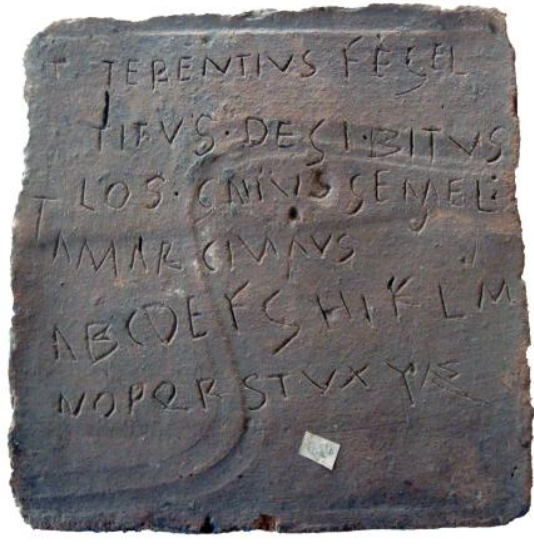

Fig. 15a. Photo de la brique de Gherla (C) Musée de Gherla inv. 656, cliché R. Zăgreanu)

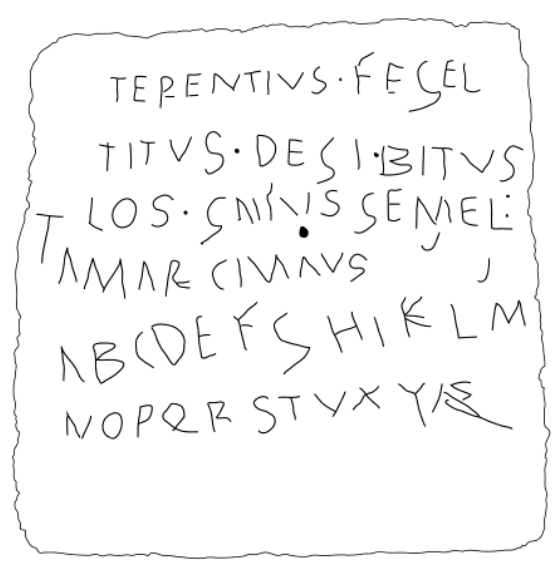

Fig. 15b. Fac-similé de la brique (D. Dana)

Transcription diplomatique :

TERENTIVS • FEGEL

TITVS $\bullet$ DEGI $\bullet$ BITVS

LOS • GAIIVS GEMEL :

$4 \Lambda \mathrm{M} \Lambda \mathrm{RCIИ} \Lambda \mathrm{VS}$

$\Lambda$ BCDEFGHIKLM

(in marg. : T)

NOPQRSTVXY .

\footnotetext{
${ }^{47}$ Faute courante de ceux qui aprennent à écrire.
} 
Ce qui donne ${ }^{48}$ :

Terentius, Fegel( ),

Titus, Degi, Bitus,

Los( ), Gaiius, Gemel( ),

$4 \quad A()$ Marci<an>us. (en marge : $T$ )

ABCDEFGHIKLM

NOPQRSTVXYZ.

Celui qui s'attela à cet exercice d'écriture ${ }^{49}$ (re)copia une liste de noms, sur quatre lignes, sans doute des soldats de l'unité en garnison à Gherla (ala II Pannoniorum $)^{50}$ - ce qui est conforté par la présence d'un nom thrace (Bitus) -, avant de reproduire, sur deux lignes, les lettres de l'alphabet latin ${ }^{51}$. La liste de possibles militaires reflète bien le mélange démographique qui caractérise l'armée romaine :

- des noms latins (qu'il s'agisse de praenomina, nomina ou cognomina), portés comme idionymes pérégrins par des provinciaux: Fegel(ius?), variante de Figel(l)ius, connu comme gentilice ${ }^{52}$; Gai\{i\}us ; Gemel(lus?); Marcianus ; Terentius; Titus.

- un nom abrégé, Los( ) ; les quelques noms en Los- enregistrés dans OPEL III 32 semblent de facture celtique ${ }^{53}$;

- un nom thrace, Bitus, qui est l'anthroponyme thrace le plus fréquent (OnomThrac 40-58);

- enfin, un nom dace ${ }^{54}$, l'un des rares noms indigènes attestés en Dacie romaine $^{55}$. Degi est une graphie du nom porté par le frère cadet du roi Décé-

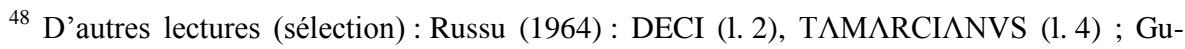
dea (1987) : Terentius fecet (1. 1), Semel (1. 3); Ardevan (2008 et Lupa) : Deci (1. 2) ; Falileyev (2009) : Degibitus ; HD : Tit<i? > us Degibitus (1. 2), Loscaiius (!) (?) (1. 3). Une autre photo sur le site du CIL : PH0009015.

${ }^{49}$ Une autre brique de Gherla comporte un exercice d'écriture ; voir Gudea 1987, 101, n 35 (dessin p. 137) ; Protase - Gudea - Ardevan 2008, 51/215, n 50 (photo et dessin Pl. VIII).

${ }^{50}$ Petolescu 2002, 74-76.

${ }^{51}$ Le meilleur parallèle en Dacie est offert par une brique d'Vlpia Sarmizegetusa, sur laquelle l'alphabet est reproduit trois fois (CIL III 8077,10 = IDR III.2 539).

${ }^{52}$ Solin - Salomies 19942, 79. Pour le passage du -i- (accentué ou non accentué) à -e-, voir Mihăescu 1978, 174-176, § 118-119.

${ }^{53}$ I. I. Russu propose $\operatorname{Los}(\mathrm{sa})$, nom celtique (ou illyrien ?).

${ }^{54}$ La lecture Degibitus de Falileyev (2009), qui en voit un nouveau nom thrace, est contredite à la fois par le point de séparation (DEGI - BITVS), le caractère exclusivement dace de degi- et l'absence de Bit(h)us dans le répertoire onomastique dace, alors que -bithus n'apparaît en seconde position qu'en Macédoine Orientale ; voir mes considérations dans OnomThrac, p. CIX. Il convient également d'écarter la correction inutile Decibitus, proposée par C. C. Petolescu, CronEpigrRom. XXX (2010), 1444 (cf. aussi $A E ́$ 2010, 1365, où il propose aux 1l. 3-4 
bale $^{56}$ : Degis chez Martial 5.3 et $\Delta$ ıñyıs chez Dion Cassius 67.7.2-3. Le même élément apparaît dans quelques noms composés daces : un roi d'époque hellé-

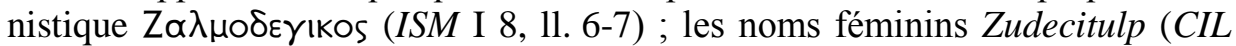
III 7481 = ISM IV 66, territoire de Tropaeum Traiani) et Zovסıєyımtous (IGBulg $\mathrm{I}^{2} 178$ bis, Odessos).

L. 4, avant Marci<an>us, il est tentant de lire un gentilice abrégé, A(elius?)/A(urelius?), ce qui nous placerait après le milieu du $\mathrm{II}^{\mathrm{e}}$ s., mais dont le rapport avec le $\mathrm{T}$ décalé à gauche est ambigu.

\section{La diffusion des « strigiles de Tarse »}

Dans la catégorie de l'instrumentum, il existe des objets qui circulent, parfois très loin, et dont la valeur augmentait progressivement. Tel devait être le cas d'un strigile de bronze $(19,5 \times 2 \mathrm{~cm})$ découvert dans une baraque du camp auxiliaire de Buciumi (dép. de Sălaj), en Dacie Porolissensis, portant la signature en grec d'un artisan de Tarse (lettres élégantes, avec des apices; epsilon, sigma et

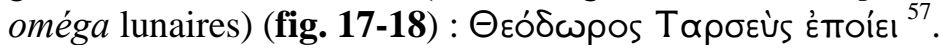

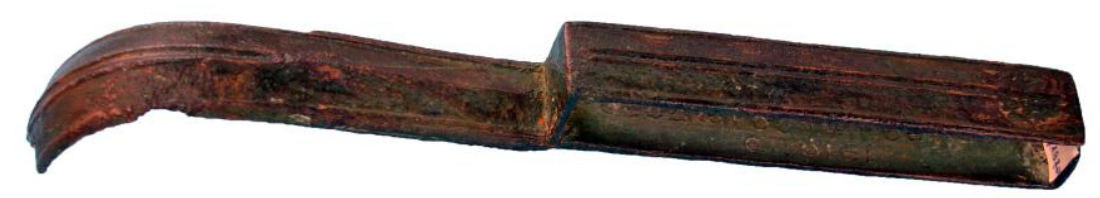

Fig. 17. Photo du strigile de Buciumi (C MJIAZ inv. c. c. 176/70, cliché D. Deac)

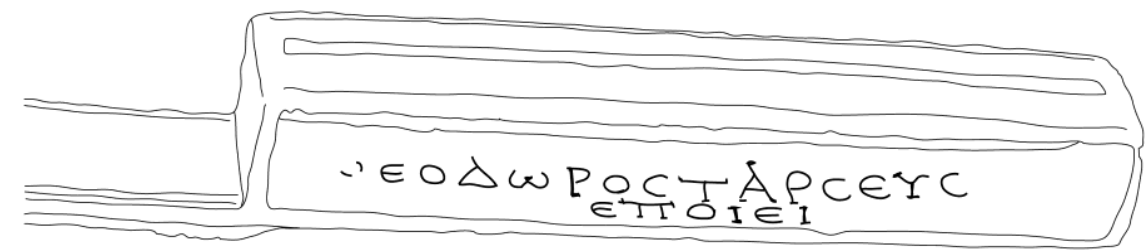

Fig. 18. Fac-similé du strigile de Buciumi (D. Dana)

Geme|l(l)a, ce qui reste très douteux; et commentaire très confus, ILD II 1007). Dans une sorte de pan-celtisme, Paki 1998, 137, $\mathrm{n}^{\circ} 25$, faisait de Desius (sic) un nom celtique.

${ }^{55}$ Voir à ce propos Dana 2004 ; Dana - Zăgreanu 2013.

${ }^{56}$ Voir Dana 2006, 113-114.

${ }^{57}$ Gudea - Lucăcel 1972, 82-83, n 1 (dessin Pl. CXIII.1) ; J. et L. Robert, BÉ, 1976, 494 ; $C I G D 39$ (Pl. X, photo) $=$ SEG LIII $746=$ ILD 648. MJIAZ (inv. c. c. 176/70). 


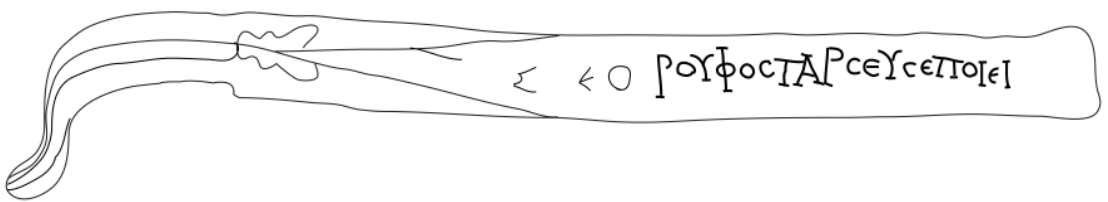

Fig. 19. Fac-similé de strigile de Vajta (D. Dana)

\section{$\odot € O \triangle W P O C T A P C \in Y C \in \Pi O$}

Fig. 20. Strigile de Mysie (AM 24, 1899, 201)

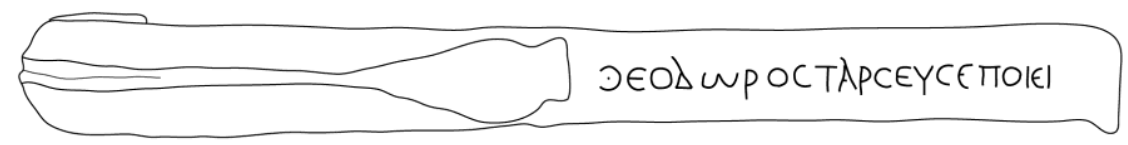

Fig. 21. Fac-similé du strigile de Trente (D. Dana)

Il est intéressant de noter un autre strigile inscrit (milieu du $\mathrm{II}^{\mathrm{e}}$-début du III s.) découvert en Pannonie Inférieure, dans un contexte archéologique qui n'est pas militaire. L'inventaire d'une tombe aristocratique à char explorée à Vajta (dép. de Fejér) livra, entre autres, un aryballe en fer et un strigile de bronze $(23,4 \mathrm{~cm})$, avec la signature d'un artisan de la même ville d'Asie Mineure

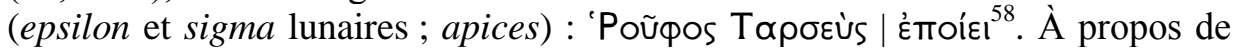
cette trouvaille pannonienne, on a parfois considéré qu'il s'agissait d'un maître itinérant. Il est néanmoins plus plausible de penser à un centre de production spécialisé au Sud de l'Asie Mineure et apprécié dans les autres provinces.

En effet, des strigiles produits par le même artisan Théodôros de Tarse sui signa l'objet retrouvé à Buciumi ont connu une diffusion large, puisque deux autres sont connus :

- ainsi, un deuxième, apparemment découvert à Ayasmat en Éolie, et apparte-

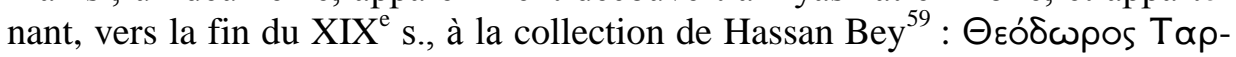

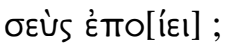

${ }^{58}$ Marosi 1939, 24 (photo Pl. II) ; Fülep 1949 ; Mócsy 1962, 686 ; Bónis 1968, 25-28 ; Bilkei $1979,33-34, \mathrm{n}^{\circ} 32$ (dessin p. 33 ; photo Pl. II.7) (= SEG XXIX 1058 ; cf. J. et L. Robert, $B E$ 1980, 336) ; Bónis 1990, 609 et Pl. 95.5 (peut-être «Wandermeister ») (cf. SEG XL 920 ; cf. M. Sève, $B E ́$ 1991, 153) ; CIGP 107 (Pl. XXII, photo et dessin) ; Mráv 2001, 38 (et cat. 105); Kovács 2007, 788 («wandering craftsman »). Musée Székesfehérvár István Király (inv. 9641). « Tarse était donc spécialiste des strigiles », notaient J. et L. Robert, $B E ́$ 1980, 336.

${ }^{59}$ Dans une collection, cf. Conze - Schuchhardt 1899, 201, $\mathrm{n}^{\circ} 1$ (avec la restitution

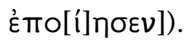


- et un troisième en Italie du Nord, récemment publié ; il fut trouvé dans une domus extra moenia de Tridentum (Trente, area di Villa Maestranzi) ${ }^{60}(21,5$

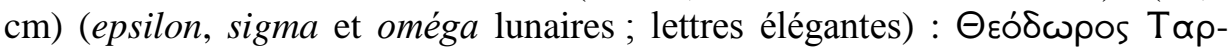

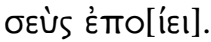

La disposition de la signature est différente sur les trois exemplaires connus, mais il s'agit du même artisan, actif au courant du $\mathrm{II}^{\mathrm{e}} \mathrm{s}$.; on remarque notamment le ductus similaire des lettres delta et alpha. Plusieurs centres de production des strigiles sont ainsi connus : en Italie, en Campanie et à Aquilée ; en Asie Mineure, à Pergame en Mysie et, comme nous venons de le voir par les signatures des deux artisans, à Tarse en Cilicie ${ }^{61}$. Au II $^{\mathrm{e}}$ s., on constate ainsi la circulation à longue distance des strigiles ciliciens, certains signés, dont un par Rufus et trois par Théodôros. La présence dans un contexte militaire n'a rien d'étonnant, puisque des études récentes, sur la foi de nombreuses découvertes, ont opportunément mis en relation la présence des balnea dans les camps avec la pratique de s'enduire d'huile parfumée et de se nettoyer à l'aide de strigiles ${ }^{62}$.

\section{Une affranchie africaine et un patron palmyrénien à Porolissum (CIL III $6249=7640$ )}

Une stèle funéraire de Porolissum, découverte il y a un siècle et demi, fut publiée dans le $C I L$, avant que sa lecture ne soit reprise à plusieurs occasions ces dernières décennies. La gravure assez maladroite, les particularités de certaines lettres, ou encore la répétition du mot liberta aux 11. 6-7, ont découragé la plupart des éditeurs, notamment pour la formule onomastique du patron. Ainsi, aux 11. 2-5, Torma lisait Victoriae | [A]fr(a)e (...) P $\Lambda \mathrm{IG} \mid \mathrm{VL} \Lambda$, tandis que von Domaszewski lisait VICTORIAI | $\Lambda$ I! FRF VIX | AN XX P- $\Lambda$ IC|VL $\Lambda$ (CIL III 7640); Gudea pat $\mid \ldots$ (1l. 4-5) ; Petruț/Zăgreanu (11. 4-5 : PAT|[---]A). Ce n'est qu'en 2014 que Ioan Piso arriva à mieux déchiffrer la formule onomastique du patron qui érigea le monument funéraire de son affranchi : P(ublius) Ae(lius) $G \mid u l a$. Il convient cependant de reprendre la lecture et l'interprétation de cette inscription qui apporte du nouveau sur le mélange démographique en Dacie romaine.

Bloc en granit rougeâtre, sculpté approximativement en forme de parallélépipède, afin d'être utilisé comme monument funéraire ; trouvé en 1864, près de la colline Pomet, probablement dans

\footnotetext{
${ }^{60}$ Bassi - Buonopane 2011, 418-419 (photos p. 420, fig. 6a-b ; dessin p. 424, Pl. 1.4) ; Buonopane 2011 (photo p. 422, fig. 8). Pour les inscriptions sur strigiles, voir Buonopane 2012.

${ }^{61}$ Voir Bolla - Buonopane 2010, 429.

${ }^{62}$ Pour les strigiles en contexte militaire (camps et sépultures), voir Beck - Chew 1991, 8183 ; sur les pratiques des militaires, moins étudiées pour des raisons idéologiques, voir Faure 2012.
} 
le cimetière romain; dans la collection de L. Szikszay, donné au musée de Zalău par le collège Wesselényi. 225 x 55 x $54 \mathrm{~cm}$. Champ épigraphique ondulé. Gravure profonde, lettres parfois maladroitement gravées (ht. $6 \mathrm{~cm}$ ). Lettres remarquables : E à une seule barre (médiane) (1. 2); après le $\mathrm{A}$ on a d'abord esquissé les lettres FR, pour les écrire plus loin (1.3); E gravé comme un $\mathrm{F}$ (1. 3) ; le second A manque de barre à la 1. 4, la lettre suivante étant probablement un E gravé comme un I ; T gravé comme un I (1. 5). Ligature CT (1. 2) ; point de séparation (1. 4). Éditions (sélection) $^{63}$ : CIL III 6249 ; Torma 1880, 129, n 5 ; CIL III 7640 ; Piso 2014, 129, n 7 (photo fig. 7) ; EHD 045100. Dans le lapidaire en plein air du Musée de Zalău (inv. C. C. 669/1988; MJIAZ inv. 261). Date : avant le milieu du $\mathrm{III}^{\mathrm{e}} \mathrm{s}$. (paléographie).

4

$D$ (is) M(anibus).

Victoriae

Afr(a)e vix(it)

an(nis) XX. P(ublius) Ae(lius?) G

ura patro-

nus liber(tae)

8

libert(a)e b(ene)

$m($ erenti).

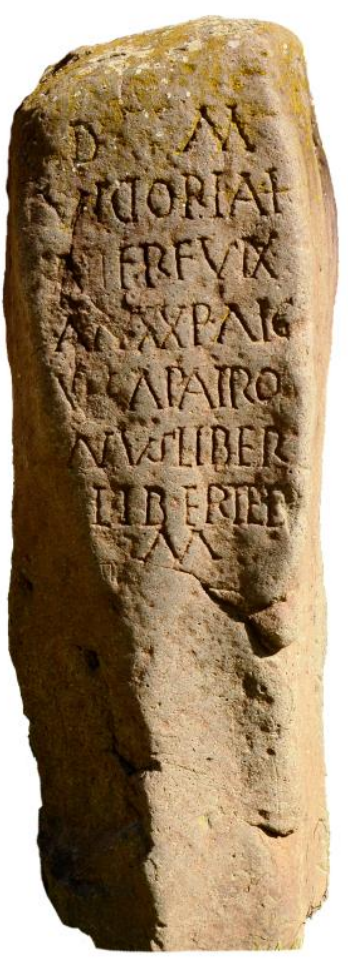

Fig. 22. Photo de l'épitaphe de Porolissum

(C MJIAZ inv. 261, cliché R. Zăgreanu)

«Le nom Victoria Afra de la défunte semble être sûr », note I. Piso, qui pense au gentilice Victorius et remarque aussitôt que «normalement le nomen de l'affranchie devrait être identique à celui de son patron, or, ici le nom de la défunte est Victoria et, si nous avons bien vu, celui de son patron est Aelius »

${ }^{63}$ Pour la bibliographie, voir la dernière édition de Piso 2014. Ajouter : Țeposu-Marinescu 1982, 105, nº 13 ; Gudea 1986, 147 ; Petruț - Zăgreanu 2011, 204, n 10 (photo p. 214, Pl. 2.10). 
(voir infra). Néanmoins, il serait plus simple de voir dans la séquence Victoria Afra l'ancien idionyme servile, suivi par l'ethnique. Cette Victoria, d'origine africaine (Afra), devait alors s'appeler après son affranchissement Aelia Victoria.

I. Piso a correctement identifié pour la première fois le prénom et le gentilice (abrégé $\mathrm{AE}$ ) du patron, partant du constat que « des problèmes pose surtout le nom de la personne qui érige le monument ». Quant au cognomen, il proposa de lire Gula. Bien que latin ("gorge, bouche »), ce nom est rarement attesté, et seulement pour des femmes ${ }^{64}$. En réalité, la photo permet de lire son cognomen autrement, car à la place du L on aperçoit un R partiellement effacé : Gura est en effet un nom sémitique bien attesté (Gura/Гoupas, Gora/Гopas), fréquent dans le milieu palmyrénien; gwr' s'explique par l'araméen («jeune animal $\gg)^{65}$. On peut donc raisonnablement penser à un soldat du numerus Palmyrenorum Porolissensium, unité présente à Porolissum, constituée à partir d'un détachement de Palmyreni sagittarii ${ }^{66}$.

En Dacie, cet anthroponyme sémitique vient donc d'être attesté pour la troisième fois, les deux autres occurrences étant :

- à Tibiscum, la stèle bilingue d'Ael. Guras $I\langle a\rangle d d e i$, [op]tio ex n(umero) Palmur(enorum) (Tibiscensium) (CIL III $7999=$ IDR III.1 154); la transcription du palmyrénien est : Gwr' Ydy hpțnn, «Gûrâ, fils de Jaddai, optio ».

- à Vlpia Sarmizegetusa, sans doute dans le même [n(umerus) PJalmyr(enorum), un certain Gora (IDR III.2 20).

Les porteurs de noms sémitiques, plus précisément palmyréniens, sont naturellement attestés à Porolissum dans le numerus ethnique, certains d'entre eux étant en outre connus comme décurions, magistrats et prêtres ${ }^{67}$. La plupart de ceux qui possèdent la citoyenneté romaine sont par ailleurs des Publii Aelii. Par

${ }^{64}$ CIL XIV 3613 ; CIL VIII 7490 (= ILA II.1 1344) et 16037 ; Audollent, DT 218.

${ }^{65}$ Grassi 2012, 47 et 182. Il existe des noms homophones dans le domaine iranien (je remercie Jean-Baptiste Yon de m'avoir transmis son étude inédite) et thrace (OnomThrac 191). 73-75.

${ }^{66}$ Petolescu 2002, 138-139 et 141-143 ; Gorea 2010 ; Țentea 2011 ; Țentea 2012, 66-71 et

${ }^{67}$ Ael. Bolhas Bannaei, vet(eranus) ex n(umero) Palmur(enorum) (CIL III 907) ; P. Aelius Iacubus, dec(urio) mun(icipii) Por(olissensis) (ILD 681); P. Ael. Malachu(s), flamen q(uin)q(uennalis) mun(icipii) S(eptimii) Por(olissensis) et sacerdos Dei n(umeri) $P$ (orolissensium) (ILD 680); [Ael.] T(h)eime[s] (Piso - Deac - Zăgreanu 2015, 224-225, $\mathrm{n}^{\circ} 16$ ) ; Ael. Thema[rsa?, vet(eranus) ex] (centurione), dec(urio) CO[---] (ILD 707); Val. Them(), vet(eranus), dec(urio) municip(ii) (ILD 688); Hamasaeus Alapatha f., Palmyr(a) (CIL XVI $68=$ IDR I 6, Palmyreni sagittarii) ; Mucianus, optio Pal(myrenorum) (ILD 690, nom d'assonance sémitique) ; Labdas Hutri (ILD 682 ; voir infra, $\mathrm{n}^{\circ}$ 12) ; Salmas Rami, ex n(umero) P(almyrenorum) (CIL III 837) ; peut-être Aurelius Manneus, princeps (CIL III 838). Voir aussi, à Augusta Traiana (Thrace), une dédicace en grec érigée par Aur. Sabinus fils de Théophilos,

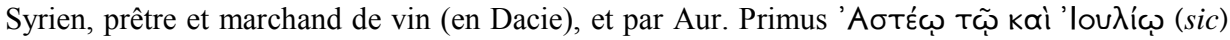

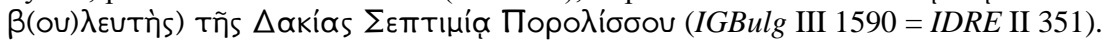


ensemble, les porteurs de noms syriens sont particulièrement visibles dans l'épigraphie de Porolissum, en rapport étroit avec la considérable présence militaire dans cet endroit stratégique. Grâce à la relecture de cette épitaphe connue depuis longtemps, on compte ainsi dans cet important site militaire du nord de la Dacie Porolissensis une esclave d'origine africaine ${ }^{68}$ et un autre militaire palmyrénien, à ajouter à la prosopographie du numerus Palmyrenorum Porolissensium.

\section{Une lettre grecque et un nom palmyrénien à Porolissum (ILD 682)}

Un autel votif de Porolissum fut publié de cette manière ${ }^{69}$ : IVPP(iteri) $M$ (aximo) $\mid$ MABIDAS HVTRI | VSLM. Son premier éditeur, N. Gudea, croyait que Iup(iteri) était une forme aberrante de Iovi. C. C. Petolescu proposa de reconnaître à la 1.1 un vocatif, Iup(iter) M(axime)!, et dans le nom du dédicant non pas un M (penché) (et par conséquent Mabidas, nom inconnu par ailleurs), mais la lettre grecque $\Sigma$, en pensant au nom $\mathrm{Zabdas}^{70}$. S. Sanie proposa à son tour la lecture M(arcus) Abidas $^{71}$, qui n'emporte pas la conviction (praenomen + cognomen). Les trois premières lettres du nom du dédicant sont ligaturées : la lettre A est encadrée par un sigma qui est très net (plutôt qu'un $\mathrm{M}$ ) et un B, chacune de ces lettres étant penchées sur l'une des hastes de A.

Autel en calcaire ( 53 × 25 × $21 \mathrm{~cm})$, avec fronton décoré d'acrotères. Découvert en 1987, lors des fouilles du camp de Pomet, près du réservoir d'eau de la praetentura sinistra, en remploi. Champ épigraphique : $40 \times 19 \mathrm{~cm}$. Ht. des lettres : $4 \mathrm{~cm}$. Ligature $\Sigma A B(1.2)$; lignes de réglage. MJIAZ (inv. 1627). Gudea 1988, 153 (dessin p. 167, fig. 7) (= $A E ́$ 1988, 976) ; C. C. Petolescu, CronEpigrRom, VIII (1988), 506 (= ILD 682); Sanie 1999, 177, $\mathrm{n}^{\circ} 4$ (dessin p. 172, fig. 1.3) (cf. $A E ́$ 1999, 1278 ; C. C. Petolescu, CronEpigrRom XIX-XX (1999-2000), 826 d ; Gudea 2007, 10, $\mathrm{n}^{\circ} 12$ (photo p. 22, fig. 11) ; Țentea 2012, 74 (Zabdas Hutri) et 149, n A.XX.4. HD 009619.

\footnotetext{
${ }^{68}$ Voir aussi, à Porolissum, l'épitaphe de Iustina Afri (ILD $706+$ CIL III $\left.7639=12544\right)$.

${ }^{69}$ Gudea 1988, 153 (et dessin p. 167, fig. 7) [= $A \dot{E}$ 1988, 976 ; l'auteur de la notice propose une faute d'écriture -u- pour -o-, donc I(ovi) Op(timo) M(aximo)]. Gudea 1999-2000, 201-202, en voit un nom d'origine hébraïque, ce qui est hasardeux. Une autre photo peut être consultée sur le site Europeana.eu.

${ }^{70}$ C. C. Petolescu, CronEpigrRom VIII (1988), 506 ; et ILD 682.

${ }^{71}$ Sanie $1999,177, \mathrm{n}^{\circ} 4$ (dessin p. 172, fig. 1.3) (cf. $A E ́$ 1999, 1278 : «cette lecture n'est pas vraisemblable ») ; et une explication du nom du dédicant par l'arabe ; C. C. Petolescu, CronEpigrRom XIX-XX (1999-2000), 826 d (qui privilégie la lecture $\Sigma$ ABIDAS, cf. Zabdas).
} 
Iup(iter?) M(aximus)

इabdas

Hutri

$4 v$ (otum) s(olvit) l(ibens) $m$ (erito).

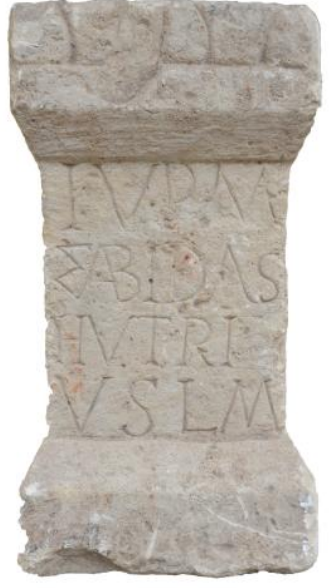

Fig. 23. Photo de la dédicace de Porolissum

(C) MJIAZ inv. 1627, cliché N. Gozman)

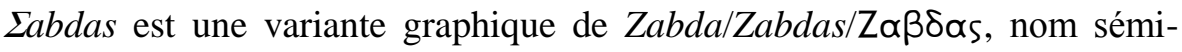
tique très fréquent, transcription de $Z b d$ ', «Don (d'un dieu) », qui est bien attesté à Palmyre et ailleurs ${ }^{72}$. Le lapicide réalisa la ligature des trois premières lettres pour que l'idionyme du dédicant puisse être gravé en entier sur une seule ligne, avec la lettre $\mathrm{D}$, au centre, de grandes dimensions ; elle a été prise par les éditeurs, à cause d'un accident récent, pour deux lettres (ID), d'où les variantes de lecture qui ont rendu sa compréhension encore plus difficile. Quant au choix du sigma, il a été sans doute déterminé par la sifflante distincte du -s- latin. On retrouve parfois ce phénomène dans l'épigraphie latine. Ainsi, à Sacidava, en

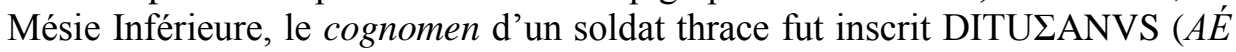
1998, 1139 = ISM IV 187), les autres graphies du nom étant Ditusanus, Dituse-

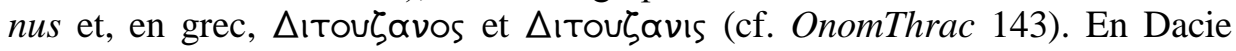
même, cet usage est mieux connu dans la transcription du toponyme d'une dédicace à Némésis pour le salut des empereurs par les curial(es) territ(orii) Euc(idavensis) (IDR II.2 $190=$ CIGD 120).

L'épitaphe et la dédicace de Porolissum discutées dans ces deux dernières notices rappellent une fois de plus la richesse des témoignages laissés par les militaires palmyréniens sur la frontière septentrionale de la Dacie romaine.

\footnotetext{
${ }^{72}$ Grassi 2012, 192.
} 
INDEX

\begin{tabular}{|c|c|}
\hline GENTILICES & Gavianus 2 \\
\hline Aelius 11 & Gemel( ) 9 \\
\hline Aurelius 2 & Germanus (eques) 4 \\
\hline Carteius 3 & $\begin{array}{l}\text { Gura } 11 \\
\text { Hutri (gén.) } 12\end{array}$ \\
\hline COGNOMINA et & $\operatorname{Los}() 9$ \\
\hline IDIONYMES & Lucius 8 \\
\hline 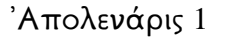 & Marcianus 9 \\
\hline $\operatorname{A\rho } \zeta \alpha K(\varepsilon) 1 \zeta 7$ & 'Poũ \\
\hline Avitus (decurio) 4 & Labdas $12(=$ Zabdas $)$ \\
\hline Bitus 9 & 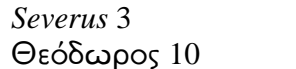 \\
\hline $\begin{array}{l}\text { Decibalus } 8 \\
\text { Degi } 9\end{array}$ & 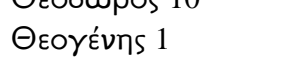 \\
\hline$\Delta$ пни́тріоs 7 & Terentius 9 \\
\hline Disala 6 & Titus 9 \\
\hline Farnaces 5 & Victoria 11 \\
\hline Fegel( ) 9 & (Zabdas) 12 \\
\hline
\end{tabular}

\author{
TOPONYMES et \\ ETHNIQUES \\ Afra 11 \\ Tapoєús 10 \\ DIVINITÉS \\ $\triangle$ eùs Nappuós 7 \\ Iup(iter) M(aximus) 12 \\ Mars 5 \\ Nymfae (sic) 8 \\ UNITÉS MILITAIRES \\ (decurio) 4 \\ (eques) 4 \\ (numerus Palmyrenorum \\ Porolissensium) 11, 12 \\ t(urma) 3 (?), 4 \\ (centuria) (?) 3
}

\author{
ABRÉVIATIONS \\ $C I G D$ \\ L. Ruscu, Corpus Inscriptionum Graecarum Dacicarum, Debrecen, \\ 2003 (Hungarian Polis Studies 10). \\ $C I G P$ \\ P. Kovács, Corpus Inscriptionum Graecarum Pannonicarum, Debrecen, \\ 2001 (Hungarian Polis Studies 8). \\ CronEpigrRom \\ C. C. Petolescu, Cronica epigrafică a României, dans Studii şi Cercetări \\ de Istorie Veche şi Arheologie (Bucarest), 1981-. \\ HD \\ Epigraphische Datenbank Heidelberg. \\ $I D R$ \\ $I D R=$ D. M. Pippidi, I. I. Russu (éds.), Inscriptiones Daciae Romanae, \\ Bucarest, 1975-. \\ IDRE \\ C. C. Petolescu, Inscriptions de la Dacie Romaine. Inscriptions externes \\ concernant l'histoire de la Dacie (I ${ }^{e r}$-III siècles), I-II, Bucarest, 1996- \\ 2000. \\ IGBulg \\ ILBulg \\ $I L D$ \\ IOSPE V \\ $I S M$ \\ G. Mihailov, Inscriptiones Graecae in Bulgaria repertae, I-V, Sofia, \\ 1958-1997. \\ B. Gerov, Inscriptiones Latinae in Bulgaria repertae [inter fluvios \\ Oescum et Iatrum], Sofia, 1989. \\ C. C. Petolescu, Inscripții latine din Dacia (ILD) I-II, Bucarest, 2005- \\ 2016. \\ A. Vinogradov, Inscriptiones antiquae orae septentrionalis Ponti Euxini \\ Graecae et Latinae. V. Byzantine Inscriptions, 2015, en ligne \\ (<http://iospe.kcl. ac.uk/corpora/byzantine/index.html>). \\ Inscriptiones Scythiae Minoris Graecae et Latinae. Inscripțiile din Scythia \\ Minor greceşti şi latine, Bucarest, 1980-.
}




$\begin{array}{ll}\text { Lupa } & \text { Vbi erat Lupa (<http://www.ubi-erat-lupa.org>). } \\ \text { MCDR } & \text { Musée de la Civilisation Dace et Romaine (Deva). } \\ \text { MJIAZ } & \text { Musée Départemental d'Histoire et d'Archéologie (Zalău). } \\ \text { MNIT } & \text { Musée National d'Histoire de la Transylvanie (Cluj). } \\ \text { MNUAI } & \text { Musée National de l'Unification (Alba-Iulia). } \\ \text { Mus. Sarmiz. } & \text { Musée de Sarmizegetusa. } \\ \text { OnomThrac } & \text { D. Dana, Onomasticon Thracicum Onomasticon Thracicum } \\ & \text { (OnomThrac). Répertoire des noms indigènes de Thrace, Macédoine } \\ & \text { Orientale, Mésies, Dacie et Bithynie, Athènes, 2014 (MELETHMATA } \\ & \text { 70). } \\ \text { OnomThracSuppl } & \text { D. Dana, Onomasticon Thracicum Supplementum, version 3.2, juillet } \\ & \text { 2016 (http://www.anhima.fr/spip.php?article1078). } \\ \text { OPEL } & \text { B. Lörincz - F. Redö (éds.), Onomasticon Provinciarum Europae Lati- } \\ & \text { narum, I-IV, Budapest-Vienne, 1994-2002 (et I', Budapest, 2005). } \\ \text { RIB } & \text { R. G. Collingwood - R. P. Wright (et R. S. O. Tomlin), The Roman } \\ & \text { Inscriptions of Britain, I-III, Oxford, 1965-2009. } \\ \text { RMD } & \text { M. M. Roxan (puis P. Holder), Roman Military Diplomas, I-V, Londres, } \\ & \text { 1978-2006. } \\ \text { SEG } & \text { Supplementum Epigraphicum Graecum, Leyden - Amsterdam, 1923-. }\end{array}$

\section{Bibliographie}

Ackner - Müller 1865 = Ackner, M. J. - Müller, Fr.: Die römischen Inschriften in Dacien. Vienne.

Ardevan - Cociş 2014 = Ardevan, A. - Cociş, S.: Drei weitere goldene Votivplättchen aus Dakien. In: Iliescu, V.- Nedu, D.- Barboş, A.-R. (éds.): Graecia, Roma, Barbaricum. In memoriam Vasile Lica. Galați, 325-328.

Avram 2013 = Avram, A.: Les Bithyniens en Thrace, en Mésie inférieure et dans le Pont Nord à l'époque impériale. In: Bru, H. - Lamarre, G. (éds.): L'Anatolie des peuples, des cités et des cultures (II millénaire av. J.-C., $V^{e}$ siècle ap. J.-C.). Colloque international de Besançon26-27 novembre 2010, t. I. Besançon, 111-132.

Bassi - Buonopane 2011 = Bassi, C. - Buonopane, A.: Un deposito di bronzi dallo scavo archeologico di un edificio di età romana a Trento, via Zanella. In: Tra protostoria e storia. Studi in onore di Loredana Capuis. Antenor Quaderni 20. Rome, 413-429.

Beck - Chew 1991 = Beck, F. - Chew, H. (éds.): Masques de fer. Un officier romain du temps de Caligula. Paris.

Bilkei 1979 = Bilkei, I.: Die griechischen Inschriften des römischen Ungarns. Alba Regia 17, 2346.

Bolla - Buonopane $2010=$ Bolla, M. - Buonopane, A.: Strigili del Museo Archeologico di Verona. Aquileia Nostra 81, 413-444.

Bónis 1968 = Bónis, É. B.: Emmaillierte Palästra-Geräte aus Brigetio. FolArch 19, 25-58.

- 1979 = Bónis, É. B.: Griechisch-römische Traditionen im Sportleben Pannoniens. Akten des XIII. internationalen Kongresses für klassische Archäologie, Berlin 1988. Mayence, 609.

Boyanov $2010=$ Boyanov, I.: Municipium Aurelianum Durostorum or vicus Gavidina. ArchBulg 14 (2), 53-59. 
Buonopane 2011 = Buonopane, A.: L'iscrizione sullo strigile. In: Bassi, C. - Buonopane, A.: Un deposito di bronzi dallo scavo archeologico di un edificio di età romana a Trento, via Zanella In: Tra protostoria e storia. Studi in onore di Loredana Capuis. Antenor Quaderni 20. Rome, 422-429.

- 2012 = Buonopane, A.: Gli strigili e le loro iscrizioni. SEBarc 10, 195-206.

Ciongradi 2009 = Ciongradi, C.: Die römische Steindenkmäler aus Alburnus Maior. Cluj.

Conze - Schuchhardt $1899=$ Conze, A. - Schuchhardt, C.: Die Arbeiten zu Pergamon 18861898. AM 24, 97-240.

Cosme 2007 = Cosme, P.: Le soldat romain entre identification et camouflage. In: Moatti, C. Kaiser, W. (éds.): Gens de passage en Méditerranée de l'Antiquité à l'époque moderne. Procédures de contrôle et d'identification. Paris, 297-312.

Cserni 1901 = Cserni, B.: Alsófehérvármegye monográphiája, II.1 (Alsófehérvármegye történelme). Nagyenyed (Aiud).

Dana 2004 = Dana, D.: Onomastique est-balkanique en Dacie romaine (noms thraces et daces). In: Ruscu, L. et alii: Orbis antiquus. Studia in honorem Ioannis Pisonis. Cluj, 430-448.

- 2006 = Dana, D.: The Historical Names of the Dacians and their Memory: New Documents and a Preliminary Outlook. Studia Universitatis Babeş-Bolyai. Historia 51 (1), 99-125.

- $2007=$ Dana, D.: Le nom du roi Décébale: aperçu historiographique et nouvelles données. In: Nemeti, S. et alii: Dacia Felix. Studia Michaeli Bărbulescu oblata. Cluj, 42-47.

- $2010=$ Dana, D.: c. r. de ILD. Studii şi Cercetări de Istorie Veche şi Arheologie 61, 201-205.

— 2011 = Dana, D.: L'impact de l'onomastique latine sur les onomastiques indigènes dans l'espace thrace. In: Dondin-Payre, M. (éd.): Les noms de personnes dans l'Empire romain. Transformations, adaptation, évolution. Scripta Antiqua 36. Bordeaux, 37-87.

- 2014a = Dana, D.: Notices onomastiques et épigraphiques I. ZPE 188, 181-198.

$-2014 b=$ Dana, D.: Notices onomastiques et épigraphiques II. ZPE 190, 149-167.

- 2014c $=$ Dana, D.: Notices onomastiques et épigraphiques (Scythie Mineure/Dobroudja) (I). Pontica 47, 465-493.

- 2016 = Dana, D.: Notices onomastiques et épigraphiques III. ZPE 198, 145-162.

Dana - Gaiu 2014 = Dana, D. - Gaiu, C.: Alte noutăți epigrafice de la Ilişua: o tessera militaris şi litere de metal descoperite în castrul auxiliar. Revista Bistriței 28, 153-163.

- 2016 = Dana, D. - Gaiu, C.: Quatre diplômes militaires du milieu du II ${ }^{\mathrm{e}}$ siècle pour l'exercitus Daciae Porolissensis trouvés à Arcobara/Ilişua (réédition). ZPE 197, 257-267.

Dana - Zăgreanu 2013 = Dana, D. - Zăgreanu, R.: Les indigènes en Dacie romaine ou la fin annoncée d'une exception: relecture de l'épitaphe CIL III 7635. Dacia NS 57, 145-159.

Eck - Pangerl 2015 = Eck, W. - Pangerl, A.: Inschriften auf metallenen militärischen Gebrauchsgegenständen. In: Henrich, P. et alii: Non solum ... sed etiam. Festschrift für Thomas Fischer zum 65. Geburtstag. Rahden, 113-126.

Falileyev 2007 = Falileyev, A.: Celtic Dacia. Personal Names, Place-Names and Ethnic Names of Celtic Origin in Dacia and Scythia Minor. Aberystwyth.

— 2009 = Falileyev, A.: A New Thracian Name from Dacia. Ad CIL III 294*. Thracia 18, 503506.

Faure 2012 = Faure, P.: Usages et images des huiles et parfums dans l'armée romaine impériale. In: Frère, D. - Hugot, L. (éds.): Les huiles parfumées en Méditerranée occidentale et en Gaule : VIII ${ }^{e}$ s. av.-VIII s. apr. J.-C. Actes du colloque, Rome, École française de Rome, du 16 au 18 novembre 2009. Naples-Rennes, 291-306.

Finály 1906 = Finály, Gy.: Római feliratos kövek Szamosújvárról. ArchÉrt 26, 37-39.

Fröhlich 1892 = Fröhlich, R.: Római feliratos tégla Szamos-Újvárból. ArchÉrt 12, 169-170.

Fülep 1949 = Fülep, I.: Rómaikori kocsitemetkezés Vajtán (Römerzeitliche Wagenbestattung in Vajta). AÉrt 76, 40-54. 
Gorea 2010 = Gorea, M.: Considérations sur la politisation de la religion à Palmyre et sur la dévotion militaire des Palmyréniens en Dacie. Semitica et Classica 3, 125-162.

Grassi 2012 = Grassi, G. F.: Semitic Onomastics from Dura Europos. The Names in Greek Script and from Latin Epigraphs. Padoue.

Gudea 1986 = Gudea, N.: Porolissum. Res publica municipii Septimii Porolissensium. Bucarest.

— 1987 = Gudea, N.: Contribuții la paleografia latină romană din Dacia. I. Inscripții pe cărămizi și țigle. Acta Musei Porolissensis 11, 91-158.

— 1988 = Gudea, N.: Castrul roman din vîrful Pomet (Porolissum). Acta Musei Porolissensis 12, $149-154$.

- 1999-2000 = Gudea, N.: Evreii în provinciile dacice. 106-275 p.Ch. EphNap 9-10, 179-208.

- 2007 = Gudea, N.: Votivdenkmäler aus Stein und ihre soziale und ethnische Wiederspiegelung. Fallstudie: Porolissum (Dacia Porolissensis). Studia Universitatis Babeş-Bolyai Theologia Catholica. Series Historia Ecclesiastica 52 (2), 7-29.

Gudea - Lucăcel 1972 = Gudea, N. - Lucăcel, V.: Das Römerlager von Buciumi. Beiträge zur Untersuchung des Limes der Dacia Porolissensis, Cluj.

Horedt 1953 = Horedt, K.: Cercetările arheologice din reg. Hoghiz-Ugra şi Teiuş. Materiale şi Cercetări Arheologice 1, 785-815.

Jajlenko 1985 = Jajlenko, V. P.: Po povodu imeni Theonoks. StudClas 23, 103.

Kovács 2002 = Kovács, P.: Greek Inscriptions in Pannonia. In: XII Congressus Internationalis Epigraphiae Graecae et Latinae: provinciae imperii Romani inscriptionibus descriptae. Barcelona, 3-8 Septembris 2002. Acta, t. I. Barcelone, 785-792.

MacMullen 1960 = MacMullen, R.: Inscriptions on Armor and the Supply of Arms in the Roman Empire. AJA 64, 23-40.

Marosi 1939 = Marosi, A.: Fejér megye népe és vallási viszonyai a római uralom idején (Die Bevölkerung und Religion von Komitat Fejér während der Römerzeit). Székesfehérvári Szemle 23, 23-37.

Matei-Popescu 2014 = Matei-Popescu, F.: Trupele auxiliare pe limesul estic al Daciei. Stadiul problemei. Angustia 17-18, 205-216.

Mihăescu 1978 = Mihăescu, H.: La langue latine dans le sud-est de l'Europe. Bucarest-Paris.

Mihăilescu-Bîrliba 2011 = Mihăilescu-Bîrliba, L.: Ex toto orbe Romano: Immigration into Roman Dacia. With Prosopographical Observations on the Population of Dacia. Colloquia Antiqua 5. Louvain-Paris-Walpole (MA).

Mitchell 2007 = Mitchell, St.: Iranian Names and the Presence of Persians in the Religious Sanctuaries of Asia Minor. In: Matthews, E. (éd.): Old and New Worlds in Greek Onomastics. Proceedings of the British Academy 148. Oxford, 151-171.

Mócsy $1962=$ Mócsy, A.: Pannonia. RE Suppl. IX, 517-776.

Moga - Piso - Drîmbărean 1998 = Moga, V. - Piso, I. - Drîmbărean, M.: Quatre monuments épigraphiques d'Apulum découverts dans le lit de Mureş. Acta Musei Napocensis 35, 109118.

Mráv 2001 = Mráv, Zs.: L'aristocratie indigène à travers les rites funéraires. In: Romans de Hongrie. $I^{e r}-V^{e}$ siècles après J.-Chr. Lyon, 30-41.

Nemeti 2005 = Nemeti, S.: Sincretismul religios în Dacia romană. Cluj.

Nuber 1972 = Nuber, H. U.: Zwei bronzene Besitzmarken aus Frankfurt/M.-Heddernheim. Zur Kennzeichung von Ausrüstungsstücken des römischen Heeres. Chiron 2, 483-507.

Oldenstein $1976=$ Oldenstein, J.: Zur Ausrüstung römischer Auxiliareinheiten. Studien zu Beschlägen und Zierat an den Ausrüstungen der römischen Auxiliareinheiten der obergermanisch-raetischen Limesgebietes aus dem zweiten und dritten Jahrhundert n. Chr. BRGK 57, 49-284. 
Ornstein 1904 = Ornstein, J.: Zur Ehrenrettung eines Fundes aus der Römerzeit. Szamosújvár/Gherla.

Paki 1989-1993 = Paki, A.: Epigraphica minora I. Acta Musei Napocensis 26-30, 291-299.

- 1998 = Paki, A.: Onomasticon Daciae (I). Die Patronymika der Provinz Dacia Porolissensis. Acta Musei Porolissensis 35, 119-146.

Petolescu 2002 = Petolescu, C. C.: Auxilia Daciae. Contribuție la istoria militară a Daciei romane. Bucarest.

Petruț - Zăgreanu 2011 = Petruț, D. - Zăgreanu, R.: The Funerary Stelae from Porolissum. Typological, Iconographical and Epigraphic Aspects. Marisia 31, 189-217.

Pfahl $2012=$ Pfahl, St. F.: Instrumenta Latina et Graca inscripta des Limesgebietes von $200 v$. Chr. bis 600 n. Chr. (ILGIL). Weinstadt.

Piso 2006 = Piso, I.: Corpus epigraphicum. In: Piso, I. (éd.): Le forum vetus de Sarmizegetusa I. Colonia Dacica Sarmizegetusa 1. Bucarest, 211-339.

— 2014 = Piso, I.: Studia Porolissensia (V). Revista Bistriței 28, 124-130.

— 2015 = Piso, I.: Studia Porolissensia (VI). Acta Musei Porolissensis 37, 193-213.

Piso - Deac - Zăgreanu 2015 = Piso, I. - Deac, D. - Zăgreanu, R.: Epigraphica Porolissensia (I). Acta Musei Porolissensis 37, 215-229.

Piso - Rusu 1990 = Piso, I. - Rusu, A.: Nympaheum-ul de la Germisara. Revista Monumentelor Istorice 59 (1), 9-17.

Popescu 2008 = Popescu, M.: La religion dans l'armée romaine de Dacie. Bucarest.

Protase 1995 = Protase, D. : L'anthroponymie thraco-dace et l'origine ethnique des porteurs dans les inscriptions de la Dacie romaine. Quelques observations. In: Frei-Stolba, R. - Herzog, H. E. (éds.): La politique édilitaire dans les provinces de l'empire romain. II ${ }^{\text {ème }}-I^{\text {ème }}$ siècles après J.-C. Berne, 157-165.

Protase - Gudea - Ardevan 2008 = Protase, D. - Gudea, N. - Ardevan, R.: Din istoria militară a Daciei romane. Castrul roman de interior de la Gherla. Aus der Militärgeschichte des römischen Dakien. Das römische Binnenkastell von Gherla. Timișoara.

Ruge 1935 = Ruge, W.: s.v. Nara (1), RE XVI.2, 1697-1698.

Rusu - Pescaru 1993 = Rusu, A. - Pescaru, E.: Germisara daco-romaine. In: Alicu, D. - Boegli, H. (éds.): La politique édilitaire dans les provinces de l'Empire Romain. I. Actes du I ${ }^{e r}$ Colloque roumano-suisse, Deva, 1991, Cluj, 201-214.

Russu 1964 = Russu, I. I.: Note epigrafice. Seria VII. Acta Musei Napocensis 1, 477-481.

Sanie 1999 = Sanie, S.: Classica et Orientalia (V). Studii şi Cercetări de Istorie Veche şi Arheologie 50 (3-4), 171-183.

Schäfer 2009 = Schäfer, A.: The Diffusion of Religious Beliefs in Roman Dacia: A Case-Study of the Gods of Asia Minor. In: Hanson, W. S. - Haynes, I. P. (éds.): The Army and Frontiers of Rome. Papers Offered to David J. Breeze on the Occasion of his Sixty-Fifth Birthday and his Retirement from Historic Scotland. Portsmouth (Rh. I.), 179-190.

Schmidt Heidenreich $2012=$ Schmidt Heidenreich, Chr.: Les inscriptions mineures dans les camps militaires romains: l'apport d'une « écriture de la marge ». In: Fuchs, M. E. et alii: Inscriptions mineures: nouveautés et réflexions. Actes du premier colloque Ductus (19-20 juin 2008, Université de Lausanne). Berne, 395-402.

Slavova 2004 = Slavova, M.: Phonology of the Greek Inscriptions in Bulgaria. Palingenesia 83. Stuttgart.

Solin 2003 = Solin, H.: Die griechischen Personennamen in Rom. Ein Namenbuch, I-III. BerlinNew York.

$-2010=$ Solin, H.: Analecta epigraphica. Arctos 44, 231-261.

Solin - Salomies $1994^{2}=$ Solin, H. - Salomies, O.: Repertorium nominum gentilium et cognominum Latinorum. Hildesheim-Zürich-New York (deuxième édition). 
Thylander $1952=$ Thylander, H.: Inscriptions du port d'Ostie. Lund.

Torma $1880=$ Torma, C.: Revidierte und neue Inschriften zu Corpus Inscriptionum Latinarum III (Dacia). AEM 4, 129-140.

Tudor 1965 = Tudor, D.: Beiträge zur Frage der Erziehung und des Unterrichts in Scythia Minor und Dacia. Das Altertum 11, 102-114.

— 1979 = Tudor, D.: Comunicări epigrafice VIII. Studii şi Cercetări de Istorie Veche şi Arheologie 30 (2), 303-310.

— 1981 = Tudor, D.: Comunicări epigrafice, IX. Studii şi Cercetări de Istorie Veche şi Arheologie 32 (3), 423-436.

Țentea 2011 = Țentea, O.: Some Remarks on Palmyreni sagittarii. On the First Records of Palmyrenes within the Roman Army. In: Piso, I. et alii: Scripta Classica. Radu Ardevan sexagenarii dedicata. Cluj, 371-377.

- 2012 = Țentea, O.: Ex Oriente ad Danubium. The Syrian Units on the Danubian Frontier of the Roman Empire. The Centre for Roman Military Studies 6. Bucarest-Cluj.

Țentea - Voişian 2014 = Țentea, O. - Voişian, V.: Băile romane de la Alburnus Maior, dealul Carpeni - edificiul E2. Raport Arheologic. Cercetări Arheologice 21, 259-289.

Țeposu-Marinescu 1982 = Țeposu-Marinescu, L.: Funerary Monuments of Dacia Superior and Dacia Porolissensis. BAR IS 128. Oxford.

Voloşciuc 2007 = Voloşciuc, A.: Scrierea cursivă în Dacia romană. Analele Banatului NS 15, 57 68.

Zgusta $1984=$ Zgusta, L.: Kleinasiatische Ortsnamen . Heidelberg.

Weber 2012 = Weber, E.: Le tavolette votive del tipo « ramo di palma ». Alcune note. In: Baratta, G. - Marengo, S. M. (éds.): Instrumenta inscripta III. Manufatti iscritti e vita dei santuari in età romana. Macerata, 247-264.

(ISSN $0418-453 \mathrm{X})$ 